\title{
Reticuloendotheliosis virus and avian leukosis virus subgroup J synergistically increase the accumulation of exosomal miRNAs
}

\author{
Defang Zhou ${ }^{1}$, Jingwen Xue ${ }^{1}$, Shuhai He ${ }^{1}$, Xusheng Du', Jing Zhou' ${ }^{1}$ Chengui Li ' Libo Huang ${ }^{1}$, \\ Venugopal Nair ${ }^{2}$, Yongxiu Yao ${ }^{2}$ and Ziqiang Cheng ${ }^{1 *}$
}

\begin{abstract}
Background: Co-infection with avian leukosis virus subgroup $J$ and reticuloendotheliosis virus induces synergistic pathogenic effects and increases mortality. However, the role of exosomal miRNAs in the molecular mechanism of the synergistic infection of the two viruses remains unknown.

Results: In this study, exosomal RNAs from CEF cells infected with ALV-J, REV or both at the optimal synergistic infection time were analysed by Illumina RNA deep sequencing. A total of 54 (23 upregulated and 31 downregulated) and 16 (7 upregulated and 9 downregulated) miRNAs were identified by comparing co-infection with two viruses, single-infected ALV-J and REV, respectively. Moreover, five key miRNAs, including miR-184-3p, miR-146a-3p, miR-146a-5p, miR-3538 and miR-155, were validated in both exosomes and CEF cells by qRT-PCR. GO annotation and KEGG pathway analysis of the miRNA target genes showed that the five differentially expressed miRNAs participated in virus-vector interaction, oxidative phosphorylation, energy metabolism and cell growth.
\end{abstract}

Conclusions: We demonstrated that REV and ALV-J synergistically increased the accumulation of exosomal miRNAs, which sheds light on the synergistic molecular mechanism of ALV-J and REV.

Keywords: Reticuloendotheliosis virus, Avian leukosis virus subgroup J, Exosomal miRNAs, Synergistic infection

\section{Background}

Viral synergism occurs commonly in nature when coinfection of two or more unrelated viruses invades the same host. Both reticuloendotheliosis virus (REV) and avian leukosis virus subgroup J (ALV-J), as two oncogenic retroviruses, consist of a set of retroviral genes, env, pol, gag and LTR, and mainly induce reticuloendotheliosis and myelocytomas, respectively $[1,2]$. Due to similar transmission routes, co-infection with ALV-J and REV can readily occur $[3,4]$ and spreads very rapidly [5-7]. Co-infection of ALV-J and REV induces more serious pathogenic effects, such as immunosuppression, growth retardation, accelerated neoplasia progression, secondary infection in chickens $[3,5]$, and increased mortality.

\footnotetext{
*Correspondence: czqsd@126.com

${ }^{1}$ College of Veterinary Medicine, Shandong Agricultural University,

Tai'an 271018, China

Full list of author information is available at the end of the article
}

Exosomes, intraluminal vesicles ranging approximately 30-100 $\mathrm{nm}$ in diameter secreted by live cells, have emerged as important molecules for intercellular communication that are involved in both normal and pathophysiological conditions, such as lactation, immune response and neuronal function, and in the development and progression of diseases, such as liver disease, neurodegenerative diseases and cancer [8-17]. Exosomes contain a wide variety of proteins, lipids, RNAs, nontranscribed RNAs, microRNAs and small RNAs to induce a diverse range of functions from intercellular communication to tumour proliferation $[14,18]$. As useful biomarkers, exosomes are also helpful for exploring the synergistic mechanisms of co-infection with ALV-J and REV.

MicroRNAs (miRNAs) constitute a large family of small noncoding RNAs functioning as major regulators of gene expression in cancer development [19-21]. The 
mature miRNA regulates spatio-temporal gene expression by binding to a seed region in the $3^{\prime}$ untranslated region (UTR) but may also bind to the $5^{\prime}$ UTR of target mRNA to enhance mRNA translation inhibition or degradation, resulting in decreased protein expression [22, 23]. Although miRNAs occupy negligible genomic space, their influence on a myriad of physiological processes, such as growth, differentiation, apoptosis, host-pathogen interactions and energy metabolism, is indubitable and relevant in tumour progression [24-30]. A growing number of studies have certified that miRNAs, including miR-122, miR-29b, miR-34a and miR-155, are key regulators of tumourigenesis, especially in viral synergistic infection [31-33]. However, the role of miRNA-mediated regulation of co-infection with ALV-J and REV remains unknown.

In the present study, to reveal the roles of miRNA profiles in the synergistic infection with ALV-J and REV, exosomal miRNAs were extracted from CEF infected with ALV-J, REV or both at the optimal synergistic infection time to analyse by Illumina RNA deep sequencing. The key miRNAs obtained from deep sequencing were validated in exosomes and CEFs by qRT-PCR. Furthermore, the affected miRNA-mRNA interactions and associated biological processes were defined by integrated target prediction analyses.

\section{Results \\ Synergistic infection of ALV-J and REV increases virus replication in CEF}

To determine the best synergistic co-infection time of ALV-J and REV in vitro, we built an in vitro model of CEFs co-infected with ALV-J and REV and conducted viral RNA transcription analysis. The qRT-PCR results showed that both ALV-J and REV RNA levels in the coinfection group were increased significantly compared to those in the single infection groups at $48 \mathrm{hpi}$ and 72 hpi and reached the highest peak at $72 \mathrm{hpi}$ (Fig. 1a and 1b). However, the ALV-J RNA levels in the co-infection group were dramatically declined compared to those in the single infection groups at $96 \mathrm{hpi}, 122 \mathrm{hpi}$ and 144 hpi (Fig. 1a) while ALV-J still synergized with REV

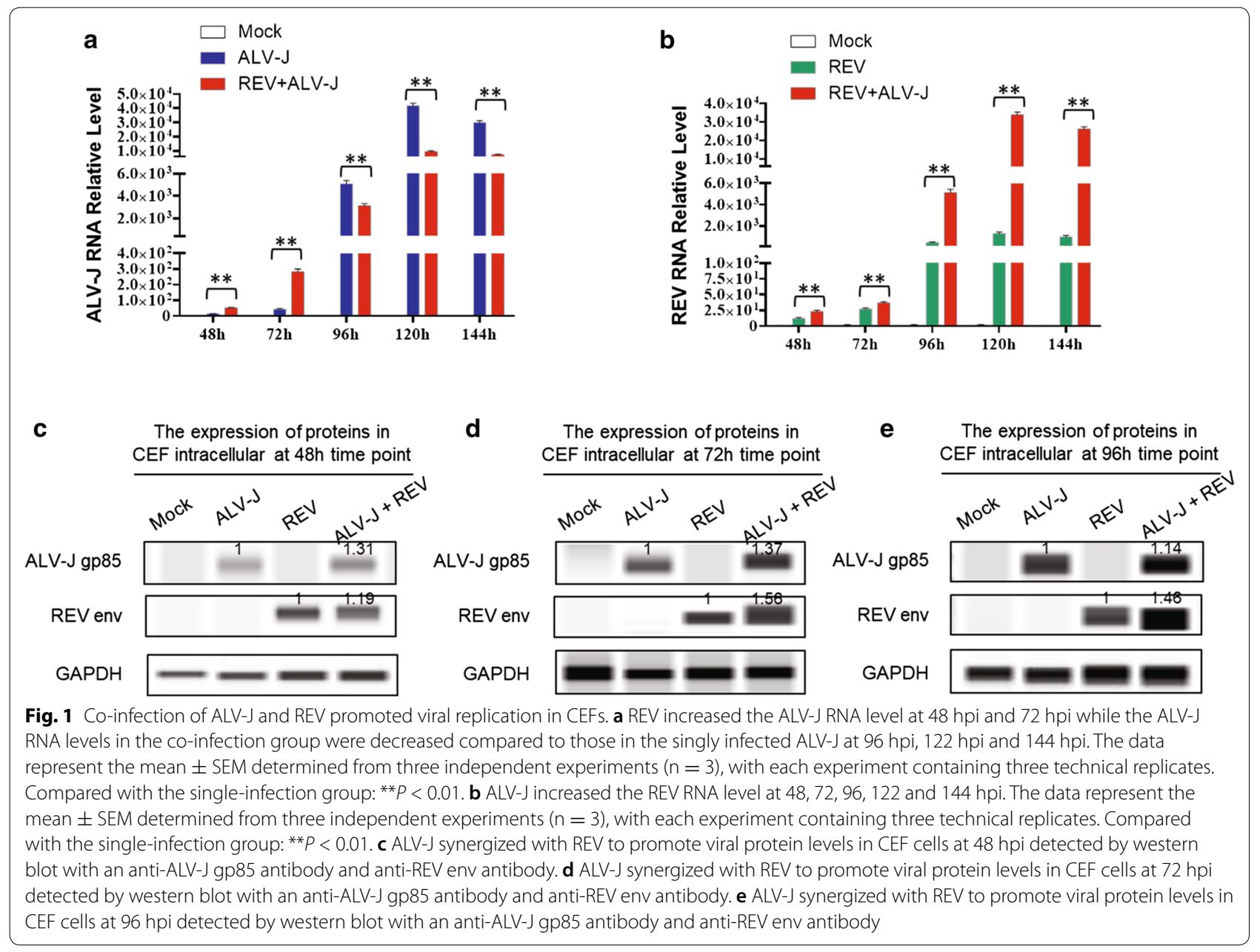


to promote viral replication at $96 \mathrm{hpi}, 120 \mathrm{hpi}$ and 144 hpi (Fig. 1b). The viral protein expression levels were detected by western blot with anti-gp85 of ALV-J or antienv of REV, and the results showed that the synergistic infection of ALV-J and REV increased each virus protein expression at $48 \mathrm{hpi}, 72 \mathrm{hpi}$ and $96 \mathrm{hpi} \mathrm{(Fig.} \mathrm{1c,} \mathrm{1d} \mathrm{and}$ 1e). Consequently, co-infection of ALV-J and REV leads to the enhancement of viral transcription and protein expression in vitro.

\section{Deep sequence analysis of exosomal miRNAs}

To explore the miRNA profile of co-infection with ALV-J and REV, the exosomal miRNA from CEFs infected with ALV-J, REV or both were analysed using miRNA wholegenome sequencing at $72 \mathrm{hpi}$. We obtained the exosomes successfully and did not have any impurities by transmission electron microscopy. The photo showed that the exosomes had the typical goblet structure, and the size varied between 40 and $150 \mathrm{~nm}$ (Fig. 2a). The purities of exosomes were also verified by western blot with
anti-Hsp70, anti-Grp78 and anti-CD81. Western blot analysis showed that both exosomal protein samples were positive for CD81, a known exosomal protein, and negative for Grp78, an endoplasmic reticulum marker (Fig. 2b).

Further, 4 miRNA libraries were generated from exosomes of normal CEFs (ExoN), CEFs infected with ALV-J (ExoJ), REV (ExoR), and both (ExoRJ), and each group had two duplicates. In total, approximately $18,482,128$ to $27,087,654$ high-quality raw reads were obtained from the exosome libraries (Table 1). The selected reads from these libraries mapped well to the chicken genome, and the perfect match rates were $42.42 \%$ and $78.75 \%$ of the total reads (Table 1). After filtering the empty adaptors, low-quality sequences and singleread sequences, almost $100 \%$ clean reads of $15-35 \mathrm{nt}$ were selected for further analysis. The remainder of the sequences were found to be other types of RNA, including rRNA, snRNA, snoRNA, tRNA, and noncoding RNA. The size distribution of small RNAs is summarized in a

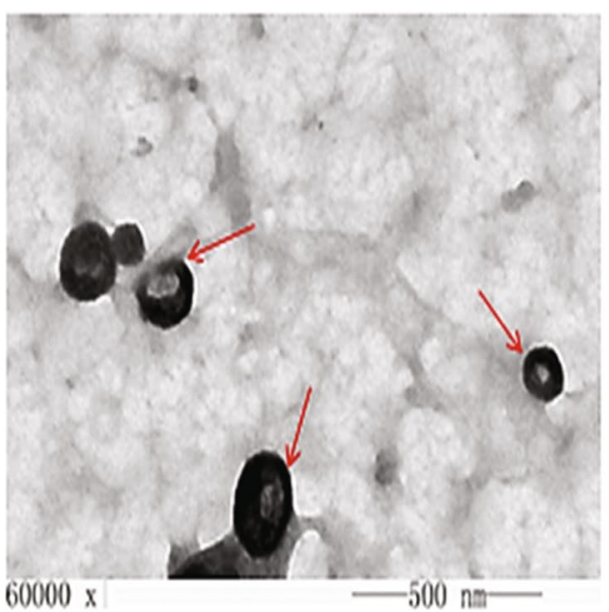

b

\section{The expression of proteins in exosomes from CEF cells}

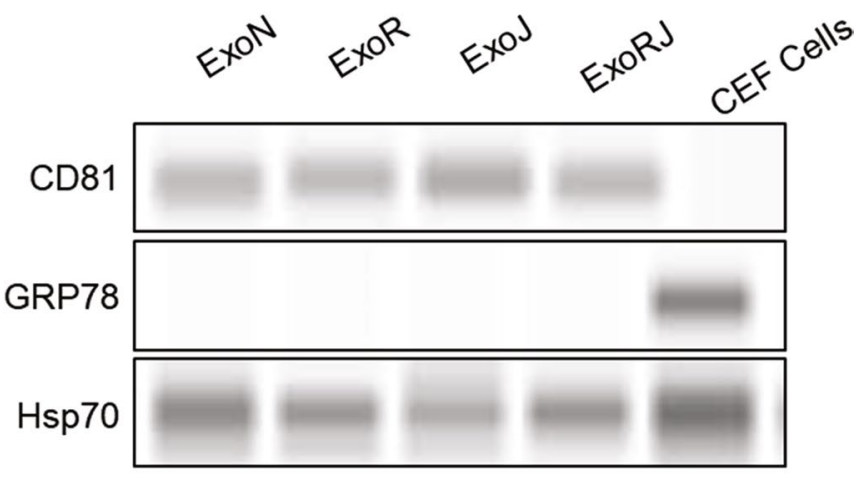

Fig. 2 Extraction and identification of exosomes from CEFs. a The morphological characterization of exosomes under electron microscopy. The arrow indicates exosomes, 40-150 nm in size. b The purities of exosomes were also verified by western blot with anti-Hsp70, anti-Grp78 or anti-CD81

Table 1 Summary of deep sequencing data for small RNAs in ExoN, ExoJ, ExoR, or ExoRJ

\begin{tabular}{|c|c|c|c|c|c|c|c|c|}
\hline Categories & ExoJ1 & ExoJ2 & ExoN1 & ExoN2 & ExoR1 & ExoR2 & ExoRJ1 & ExoRJ2 \\
\hline Raw reads & $32,802,706$ & $33,930,695$ & $34,958,139$ & $32,703,162$ & $40,626,293$ & $39,142,674$ & $35,806,903$ & $47,791,565$ \\
\hline Clean reads & $25,528,138$ & $22,734,959$ & $18,482,128$ & $25,269,659$ & $26,451,518$ & $19,409,373$ & $27,087,654$ & $26,375,518$ \\
\hline Map to genome percent (\%) & 72.43 & 77.02 & 67.15 & 78.75 & 61.58 & 58.72 & 44.3 & 42.42 \\
\hline Exon-antisense & 993,966 & $1,018,809$ & 418,741 & 761,889 & 516,146 & 323,086 & 414,847 & 346,494 \\
\hline Intron-sense & 82,595 & 67,299 & 67,235 & 94,664 & 78,494 & 59,812 & 75,956 & 60,438 \\
\hline Intron-antisense & 66,891 & 66,383 & 48,160 & 84,863 & 56,699 & 33,887 & 63,692 & 54,035 \\
\hline miRNAs & $5,564,323$ & $5,391,541$ & $3,120,429$ & $3,314,531$ & $1,154,633$ & 583,363 & $1,048,558$ & 714,469 \\
\hline
\end{tabular}


Fig. 3. The results showed that the peaks in CEF-uninfected, infected ALV-J, REV, or both were concentrated at $21-23 \mathrm{nt}$.

\section{Identification of ALV-J and REV synergistic activated exosomal miRNAs in CEFs}

To compare the differentially expressed miRNAs between co-infected and the two single virus infected CEFs, the differentially expressed miRNAs were mapped to the miRNA precursors of the reference species in the Sanger miRBase 21.0 database [34]. Using a P-value <
0.05 and $\mid \log 2$ (fold change) $\mid \geq 1$ as the cut-off values, a total of 54 (23 upregulated and 31 downregulated) and 16 (7 upregulated and 9 downregulated) miRNA genes were identified by comparing ExoRJ with ExoJ and ExoR, respectively (Fig. 4, Tables 2,3 ).

\section{Validation of miRNA expression using qRT-PCR}

To validate the results of deep sequencing, five miRNAs, including miR-184-3p, miR-146a-3p, miR-146a-5p, miR-3538 and miR-155 that changed significantly in the co-infection group compared to each single infection
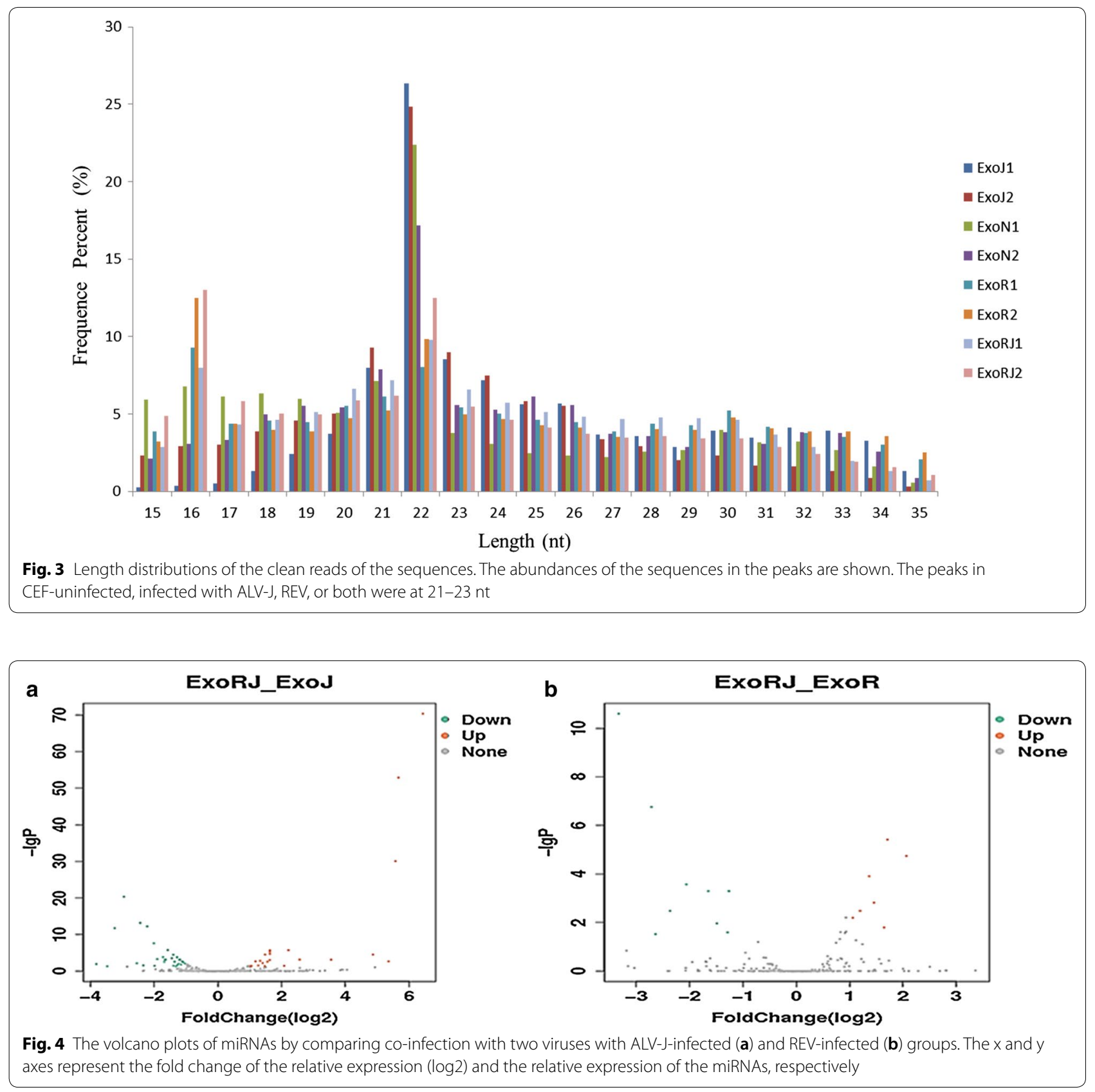


\begin{tabular}{|c|c|c|c|c|}
\hline miRNA name & $\mathrm{FC}$ & $P$ value & FDR & Up/down \\
\hline gga-miR-92-3p & 2.77 & $4.45 \times 10^{-3}$ & 0.04 & Up \\
\hline gga-miR-456-3p & 2.8 & $9.21 \times 10^{-7}$ & $3.18 \times 10^{-5}$ & Up \\
\hline gga-miR-429-3p & 86 & $8.65 \times 10^{-74}$ & $4.47 \times 10^{-71}$ & Up \\
\hline gga-miR-375 & 29.1 & $8.16 \times 10^{-7}$ & $3.02 \times 10^{-5}$ & Up \\
\hline gga-miR-3538 & 3.1 & $4.48 \times 10^{-7}$ & $1.78 \times 10^{-5}$ & Up \\
\hline gga-miR-3529 & 3.08 & $5.52 \times 10^{-5}$ & $1.06 \times 10^{-3}$ & Up \\
\hline gga-miR-30c-2-3p & 2.05 & $4.18 \times 10^{-3}$ & 0.04 & Up \\
\hline gga-miR-30a-3p & 3.1 & $1.04 \times 10^{-7}$ & $4.47 \times 10^{-6}$ & Up \\
\hline gga-miR-223 & 2.06 & $3.87 \times 10^{-3}$ & 0.039 & Up \\
\hline gga-miR-222b-3p & 11.8 & $3.48 \times 10^{-5}$ & $7.50 \times 10^{-4}$ & Up \\
\hline gga-miR-221-3p & 4.65 & $3.67 \times 10^{-8}$ & $1.89 \times 10^{-6}$ & Up \\
\hline gga-miR-2131-3p & 2.66 & $4.54 \times 10^{-4}$ & $6.35 \times 10^{-3}$ & Up \\
\hline gga-miR-184-3p & 5.94 & $2.85 \times 10^{-5}$ & $7.01 \times 10^{-4}$ & Up \\
\hline gga-miR-1684a-3p & 2.51 & $8.90 \times 10^{-5}$ & $1.64 \times 10^{-3}$ & Up \\
\hline gga-miR-155 & 50.8 & $5.03 \times 10^{-56}$ & $1.30 \times 10^{-53}$ & Up \\
\hline gga-miR-146a-5p & 41 & $1.37 \times 10^{-4}$ & $2.37 \times 10^{-3}$ & Up \\
\hline gga-miR-146a-3p & 47.7 & $5.35 \times 10^{-33}$ & $9.22 \times 10^{-31}$ & Up \\
\hline gga-miR-144-3p & 2.95 & $1.79 \times 10^{-4}$ & $2.72 \times 10^{-3}$ & Up \\
\hline gga-miR-142-5p & 2.27 & $1.33 \times 10^{-4}$ & $2.37 \times 10^{-3}$ & Up \\
\hline gga-miR-1416-5p & 4.24 & $3.94 \times 10^{-3}$ & 0.039 & Up \\
\hline gga-miR-1329-5p & 2.41 & $2.72 \times 10^{-3}$ & 0.03 & Up \\
\hline gga-let-7c-5p & 2.06 & $3.45 \times 10^{-3}$ & 0.036 & Up \\
\hline gga-miR-6548-5p & 0.071 & $9.08 \times 10^{-4}$ & 0.011 & Down \\
\hline gga-miR-460a-3p & 0.382 & $3.29 \times 10^{-3}$ & 0.035 & Down \\
\hline gga-miR-455-5p & 0.336 & $3.03 \times 10^{-8}$ & $1.74 \times 10^{-6}$ & Down \\
\hline gga-miR-455-3p & 0.38 & $1.13 \times 10^{-6}$ & $3.64 \times 10^{-5}$ & Down \\
\hline gga-miR-34a-3p & 0.322 & $3.22 \times 10^{-5}$ & $7.24 \times 10^{-4}$ & Down \\
\hline gga-miR-32-3p & 0.172 & $5.48 \times 10^{-4}$ & $7.45 \times 10^{-3}$ & Down \\
\hline gga-miR-30e-5p & 0.305 & $4.99 \times 10^{-6}$ & $1.51 \times 10^{-4}$ & Down \\
\hline gga-miR-30d & 0.45 & $1.44 \times 10^{-3}$ & 0.017 & Down \\
\hline gga-miR-30b-5p & 0.215 & $6.92 \times 10^{-15}$ & $5.96 \times 10^{-13}$ & Down \\
\hline gga-miR-301b-3p & 0.434 & $3.98 \times 10^{-5}$ & $8.02 \times 10^{-4}$ & Down \\
\hline gga-miR-301a-3p & 0.487 & $6.54 \times 10^{-4}$ & $8.68 \times 10^{-3}$ & Down \\
\hline gga-miR-2954 & 0.185 & $6.53 \times 10^{-16}$ & $6.75 \times 10^{-14}$ & Down \\
\hline gga-miR-26a-3p & 0.199 & $2.51 \times 10^{-3}$ & 0.028 & Down \\
\hline gga-miR-219b & 0.472 & $2.5 \times 10^{-4}$ & $3.63 \times 10^{-3}$ & Down \\
\hline gga-miR-2131-5p & 0.412 & $5.73 \times 10^{-6}$ & $1.65 \times 10^{-4}$ & Down \\
\hline gga-miR-20a-3p & 0.253 & $3.68 \times 10^{-3}$ & 0.038 & Down \\
\hline gga-miR-203a & 0.09 & $5.58 \times 10^{-3}$ & 0.049 & Down \\
\hline gga-miR-199-5p & 0.313 & $1.88 \times 10^{-4}$ & $2.78 \times 10^{-3}$ & Down \\
\hline gga-miR-193b-3p & 0.13 & $3.44 \times 10^{-23}$ & $4.45 \times 10^{-21}$ & Down \\
\hline gga-miR-190a-5p & 0.268 & $2.00 \times 10^{-5}$ & $5.18 \times 10^{-4}$ & Down \\
\hline gga-miR-18a-5p & 0.465 & $1.66 \times 10^{-4}$ & $2.69 \times 10^{-3}$ & Down \\
\hline gga-miR-181a-3p & 0.439 & $3.01 \times 10^{-5}$ & $7.08 \times 10^{-4}$ & Down \\
\hline gga-miR-1729-5p & 0.106 & $2.66 \mathrm{E}-14$ & $1.99 \times 10^{-12}$ & Down \\
\hline gga-miR-16-5p & 0.414 & $2.19 \times 10^{-3}$ & 0.025 & Down \\
\hline gga-miR-148a-5p & 0.462 & $1.64 \times 10^{-4}$ & $2.69 \times 10^{-3}$ & Down \\
\hline gga-miR-146c-5p & 0.317 & $4.03 \times 10^{-5}$ & $8.02 \times 10^{-4}$ & Down \\
\hline gga-miR-146c-3p & 0.404 & $5.18 \times 10^{-3}$ & 0.0461 & Down \\
\hline gga-miR-146b-5p & 0.368 & $1.09 \times 10^{-5}$ & $2.97 \times 10^{-4}$ & Down \\
\hline
\end{tabular}

Table 2 (continued)

\begin{tabular}{lllll}
\hline miRNA name & FC & P value & FDR & Up/down \\
\hline gga-miR-1451-5p & 0.249 & $3.64 \times 10^{-10}$ & $2.35 \times 10^{-8}$ & Down \\
gga-miR-10b-3p & 0.426 & $8.66 \times 10^{-4}$ & 0.011 & Down \\
gga-miR-101-2-5p & 0.39 & $1.76 \times 10^{-4}$ & $2.72 \times 10^{-3}$ & Down \\
\hline
\end{tabular}

FC fold change, FDR false discovery rate (corrected $P$ value)

\begin{tabular}{lllll}
$\begin{array}{l}\text { Table 3 Differentially } \\
\text { between ExoRJ and ExoR }\end{array}$ & expressed & microRNAs \\
\hline miRNA name & FC & p value & FDR & Up/down \\
\hline gga-miR-146a-3p & 4.18 & $1.66 \times 10^{-7}$ & $1.84 \times 10^{-5}$ & Up \\
gga-miR-155 & 3.27 & $2.62 \times 10^{-8}$ & $3.87 \times 10^{-6}$ & Up \\
gga-miR-184-3p & 3.13 & $5.41 \times 10^{-4}$ & $1.59 \times 10^{-2}$ & Up \\
gga-let-7a-2-3p & 2.74 & $3.08 \times 10^{-5}$ & $1.51 \times 10^{-3}$ & Up \\
gga-miR-458a-3p & 2.29 & $8.31 \times 10^{-5}$ & $3.34 \times 10^{-3}$ & Up \\
gga-miR-429-3p & 2.08 & $1.89 \times 10^{-4}$ & $6.44 \times 10^{-3}$ & Up \\
gga-miR-3538 & 0.42 & $9.19 \times 10^{-6}$ & $5.08 \times 10^{-4}$ & Down \\
gga-miR-1454 & 0.41 & $1.04 \times 10^{-3}$ & $2.56 \times 10^{-2}$ & Down \\
gga-miR-460b-5p & 0.36 & $3.45 \times 10^{-4}$ & $1.09 \times 10^{-2}$ & Down \\
gga-miR-133c-3p & 0.32 & $8.86 \times 10^{-6}$ & $5.08 \times 10^{-4}$ & Down \\
gga-miR-489-3p & 0.24 & $3.68 \times 10^{-6}$ & $2.71 \times 10^{-4}$ & Down \\
gga-miR-499-5p & 0.19 & $7.76 \times 10^{-5}$ & $3.34 \times 10^{-3}$ & Down \\
gga-miR-1677-3p & 0.16 & $1.36 \times 10^{-3}$ & $3.01 \times 10^{-2}$ & Down \\
gga-miR-1563 & 0.15 & $7.89 \times 10^{-10}$ & $1.75 \times 10^{-7}$ & Down \\
gga-miR-206 & 0.1 & $5.71 \times 10^{-14}$ & $2.53 \times 10^{-11}$ & Down \\
\hline
\end{tabular}

FC fold change, FDR false discovery rate (corrected $P$ value)

group, were selected for qRT-PCR analysis with primers in Table 4. After RNA was isolated from ExoN, ExoJ, ExoR and ExoRJ at $72 \mathrm{hpi}$, all 5 miRNAs showed expression profiles in CEF exosomes that were consistent with the small RNA sequencing data (Fig. 5a). Furthermore, the expressions of these five miRNAs were also verified in CEF cells co-infected with ALV-J and REV $72 \mathrm{hpi}$. Although the variation trends of miR-184-3p, miR146a-3p, miR-3538 and miR-155 in both CEF cells and exosomes were consistent, some changes in each miRNA in CEF cells were less than in exosomes, indicating that the exosomes stably maintained these miRNAs (Fig. 5b).

\section{Target prediction}

To understand the biological functions of the miRNAs identified in our analysis, miRanda was used to predict targets of the differentially expressed miRNA. Numerous target genes, 19,450 and 6058 for 54 and 16 miRNAs, respectively, were predicted as potential miRNA targets (Additional files 1 and 2). A GO annotation of the predicted target genes revealed that 100 and 35 target genes were significantly annotated for the 54 and 16 miRNAs, respectively, and they were involved in cellular processes, 
Table 4 Primers used to detect miRNA expression using qRT-PCR

\begin{tabular}{lll}
\hline miRNA name & Mature sequences & Primer $\left(\mathbf{5}^{\prime} \mathbf{- 3} \mathbf{3}^{\prime}\right)$ \\
\hline gga-miR-146a-5p & UGAGAACUGAAUUCCAUGGGUU & GTGAGAACTGAATTCCATGGGTT \\
gga-miR-146a-3p & ACCCAUGGGCUCAGUUCUUCAG & ACCCATGGGGCTCAGTTCTTC \\
gga-miR-184-3p & UGGACGGAGAACUGAUAAGGGU & TGGACGGAGAACTGATAAGGGT \\
gga-miR-3538 & GUUCGGUGAUGAAACCAUGGA & GGTTCGGTGATGAAACCATGGA \\
gga-miR-155 & UUAAUGCUAAUCGUGAUAGGG & GGTTAATGCTAATCGTGATAGGG \\
U6-F & & CTCGCTTCGGCAGCACA \\
U6-R & & AACGCTTCACGAATTTGCGT \\
\hline
\end{tabular}

immune system processes, biology regulation, such as cytoskeleton organization, regulation of Ras protein signal transduction, ATP binding and guanyl-nucleotide exchange factor activity (Fig. 6, Additional files 3 and 4). To further analyse the roles that these miRNAs might play in regulatory networks, the putative miRNA targets were assigned to KEGG pathways using the KEGG GENES Database, PATHWAY Database and LIGAND Database. The results indicated that the most abundant KEGG terms were involved in the Toll-like receptor signalling pathway, oxidative phosphorylation, ribosome and other biological processes (Fig. 7, Additional files 5 and 6). In summary, these findings demonstrated that the differentially expressed miRNAs play important regulatory roles in virus-vector interaction, energy metabolism and cell growth.

\section{Discussion}

In this study, an enhancement of viral transcription and protein expression was observed in ALV-J and REV coinfected CEF cells and reached a peak at $72 \mathrm{hpi}$. After Illumina small RNA deep sequencing of exosomes from CEFs co-infected with ALV-J and REV, a total of 54 (23 upregulated and 31 downregulated) and 16 (7 upregulated and 9 downregulated) miRNAs were identified by comparing the significantly differentially expressed miRNAs of ExoRJ with ExoJ and ExoR, respectively. Further, 5 miRNAs, including miR-184-3p, miR-146a-3p, miR146a-5p, miR-3538 and miR-155, were verified by qRTPCR and found to be consistent with the sequencing analysis. The analysis of the target prediction data demonstrated that these differentially expressed miRNAs participated in aspects of virus-vector interaction, oxidative
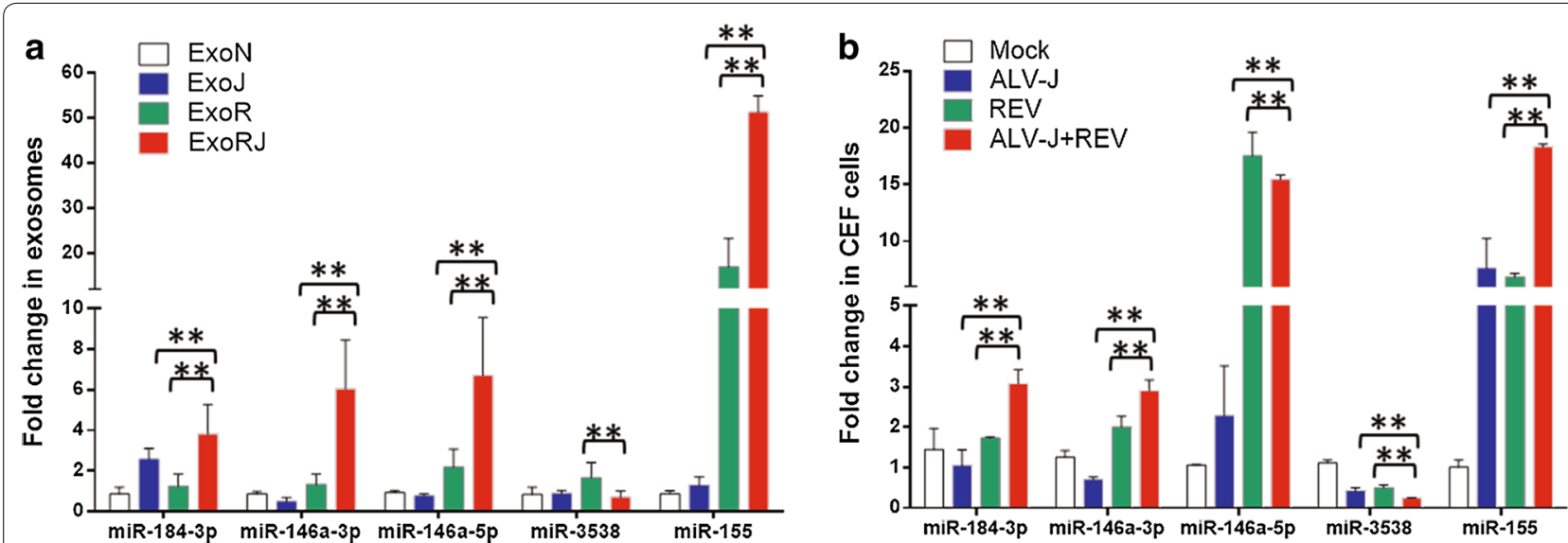

Fig. 5 The qRT-PCR analysis for five miRNAs from exosomes and CEF cells. a The qRT-PCR results of the five miRNAs in exosomes were consistent with the sequencing. The data represent the mean \pm SEM determined from three independent experiments $(n=3)$, with each experiment containing three technical replicates. Compared with the single-infection group: ${ }^{* *} P<0.01$. $\mathbf{b}$ The qRT-PCR results of four miRNAs in CEFs, including miR-184-3p, miR-146a-3p, miR-3538 and miR-155, were consistent with that in exosomes while miR-146a-5p expression in singly infected REV was significantly higher than co-infection with two viruses. The data represent the mean \pm SEM determined from three independent experiments $(n=$ 3), with each experiment containing three technical replicates. Compared with the single-infection group: ${ }^{* *} P<0.01$ 


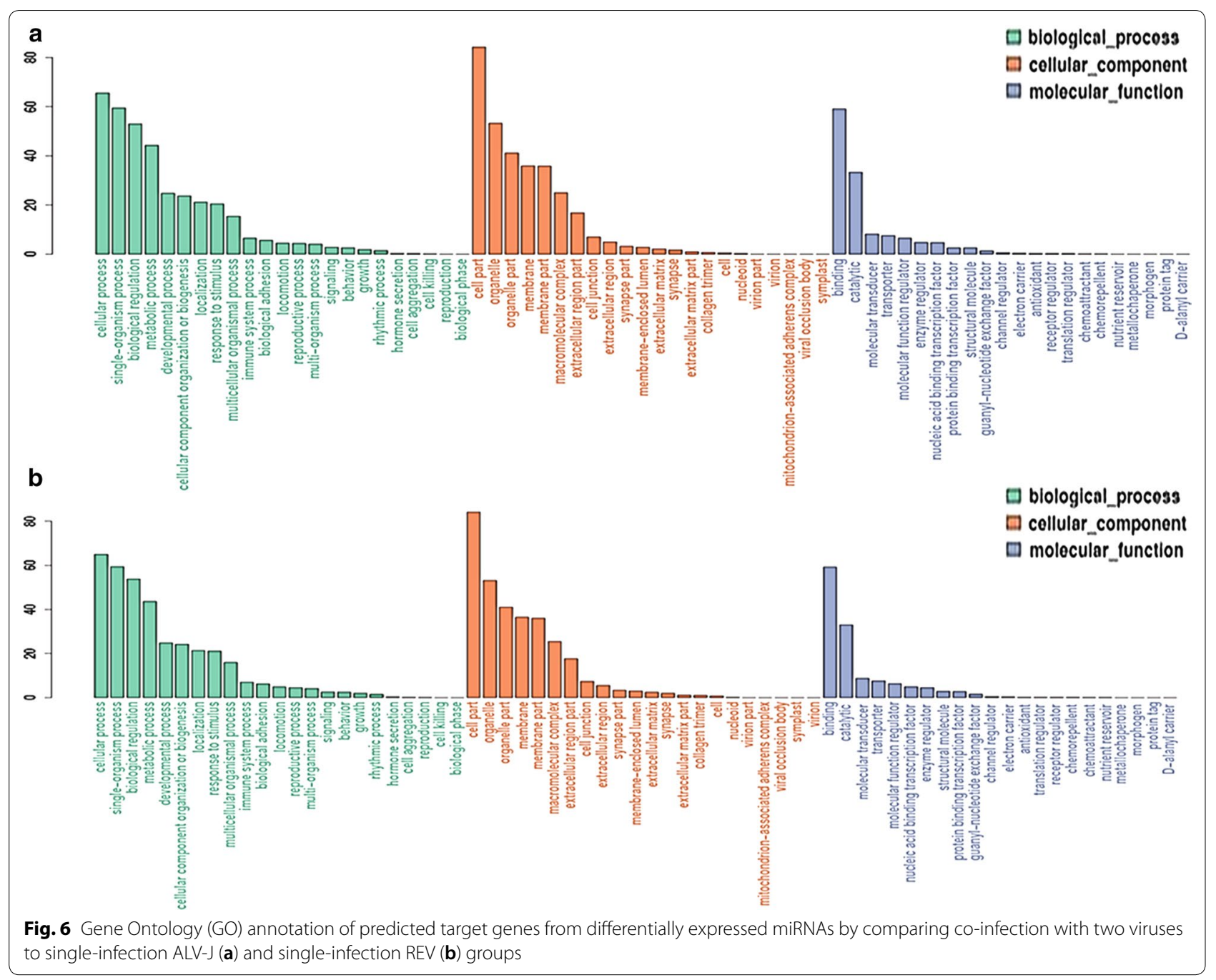

phosphorylation, energy metabolism and cell growth in the process of co-infection with ALV-J and REV.

Useful as a biomarker, exosomes are overproduced by most proliferating cell types and contain a wide variety of microRNAs to induce a diverse range of functions, such as antigen presentation, cellular responses to environmental stresses, and propagation of pathogens [35-39]. Some miRNAs, such as let-7, miR-1, miR-15, miR-16, miR-151 and miR-375, which have roles in angiogenesis, haematopoiesis, exocytosis and tumourigenesis, have been reported in exosomes [40-44]. To further understand the relationship between exosomes and the parental CEF cells, miR-184-3p, miR-146a-3p, miR-146a-5p, miR-3538 and miR-155 expression was verified in both exosomes and CEF cells. In addition to miR-146a-5p, the qRT-PCR results in CEF cells of miR-184-3p, miR146a-3p, miR-3538 and miR-155 were consistent with that in exosomes, suggesting that these miRNAs are key regulators in co-infection with ALV-J and REV.
In tumour progression, especially induced by viral synergistic infection, several studies have verified that miRNAs influence growth, differentiation, apoptosis, host-pathogen interactions, energy metabolism and other physiological processes [31-33]. While infecting cells, viruses integrate into the host genome to ensure viral persistence, which requires certain conditions for virus-vector interaction [45]. Simultaneously, the viral replication also benefits the cell's transcriptional and translational machinery, which may enhance the growth of the host cells. In addition, these regulations are based on energy metabolism [46, 47]. Our data suggested that the significantly differentially expressed miRNAs between co-infected and two single virus infected CEFs participated in energy metabolism, virus-vector interaction and cell growth, suggesting that these miRNAs are key regulators in co-infection with ALV-J and REV. These initial findings will lead to further exploration of the mechanism of ALV-J synergistic infection with REV. 


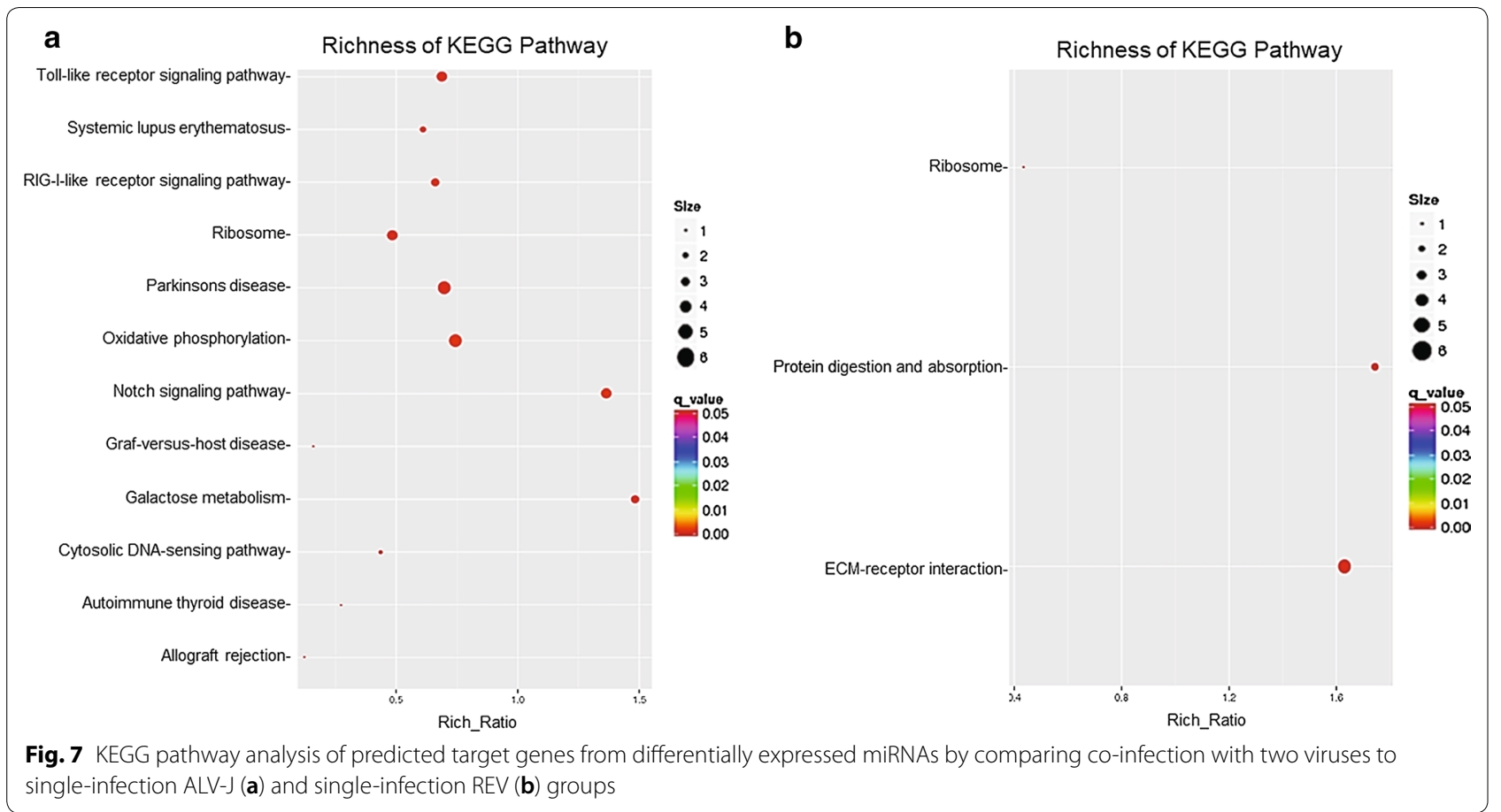

\section{Conclusion}

We demonstrated that REV and ALV-J synergistically increased the accumulation of exosomal miRNAs. We revealed that a total of 54 and 16 miRNA genes were identified by comparing co-infection with two viruses with single-infected ALV-J and REV, respectively. These differentially expressed miRNAs participated in virusvector interaction, oxidative phosphorylation, energy metabolism and cell growth, indicating potential new avenues to study the mechanism of synergistic infection of ALV-J and REV.

\section{Methods}

\section{Cells and virus}

DF-1 and chicken embryo fibroblasts (CEFs) cells were maintained in Dulbecco's modified Eagle's medium (DMEM) supplemented with $10 \%$ foetal bovine serum (FBS), 1\% penicillin/streptomycin, and 1\% l-glutamine, in a $5 \% \mathrm{CO}_{2}$ incubator at $37^{\circ} \mathrm{C}$. The stock SNV strain of REV at $10^{3.2} 50 \%$ tissue culture infectious doses $\left(\mathrm{TCID}_{50}\right)$ and NX0101 strain of ALV-J at $10^{3.8} \mathrm{TCID}_{50}$ were maintained in our laboratory. The TCID 50 of the SNV and NX0101 strains were titrated by limiting dilution in DF-1 culture.

\section{Extraction of exosomes from CEF cells}

The exosomes from Mock, single infection of ALV-J or REV, or co-infection of both ALV-J and REV CEFs were isolated using Total Exosome Isolation Reagent
(Thermo Fisher Scientific) based on the manufacturer's instructions.

\section{Transmission electron microscopy}

The protocol was conducted as described in a previous study [48].

\section{Western blotting}

CEF cells were lysed in cell lysis buffer (Beyotime) and incubated on ice for 5 minutes. ALV-J gp85, REV env expression, Hsp70, GRP78 and CD81 were detected by simple western analysis with anti-NX0101 gp85, antiSNV env antibody, anti-Hsp70 (Bioss), anti-GRP78 (Bioss) antibody and anti-CD81 (Bioss) antibody at a 1:200, 1:200, 1:1000, 1:1000 and 1:1000 dilution, respectively.

\section{Illumina small RNA deep sequencing}

Total RNA of the infected CEF exosome samples was separated by $15 \%$ agarose gels to extract the small RNA (18-30 nt). After precipitation by ethanol and centrifugal enrichment of the small RNA population, the library was prepared according to the method and process of the Small RNA Sample Preparation Kit (Illumina, RS-200-0048). The RNA concentration of the library was measured using Qubit ${ }^{\circledR}$ RNA Assay Kit in Qubit $^{\circledR} 2.0$ to preliminarily quantify and then dilute to 
$1 \mathrm{ng} / \mu \mathrm{l}$. The insert size was assessed using the Agilent Bioanalyzer 2100 system (Agilent Technologies, CA, USA). The library with the expected insert size was then quantified accurately using TaqMan fluorescence probes of the AB Step One Plus Real-Time PCR system (library valid concentration $>2 \mathrm{nM}$ ). The qualified libraries were sequenced by an Illumina HiSeq 2500 platform and $50 \mathrm{bp}$ single-end reads were generated.

\section{Real-time quantitative reverse transcription polymerase chain reaction}

Total RNA from CEF exosomes of either Mock, single infection of ALV-J or REV, or co-infection of both ALV-J and REV were isolated using the Tiangen RNeasy mini kit according to the manufacturer's instructions, with optional on-column DNase digestion. RNA integrity and concentration were assessed by agarose gel electrophoresis and spectrophotometry. RNA (1 $\mu \mathrm{g}$ per triplicate reaction) was reverse transcribed to cDNA using the TaqMan Gold Reverse Transcription kit (Applied Biosystems). Real-time RT-PCR (qRT-PCR) was carried out using SYBR ${ }^{\circledR}$ Premix Ex TaqTM, and ALV-J or REV specific primers (Table 5). All values were normalized to the endogenous control GAPDH to control for variation. For qRT-PCR of miR-184-3p, miR-146a-3p, miR-146a-5p, miR-3538 and miR-155, we used a miRcute miRNA first-stand cDNA synthesis kit and a miRcute miRNA qPCR detection kit (SYBR Green) (TIANGEN). The reverse primer was provided in the miRcute miRNA qPCR detection kit as a primer complementary to the poly (T) adapter. Data were collected on an ABI PRISM 7500 and analysed via Sequence Detector v1.1 software. All values were normalized to the endogenous control U6 to control for variation. The specific primer for U6 was described in Table 4. Assays were performed in triplicate and average threshold cycle (CT) values were used to determine relative concentration differences based on the $\Delta \triangle \mathrm{CT}$ method of relative quantization described in the manufacturer's protocol.

Table 5 Primers used for real-time PCR

\begin{tabular}{llll}
\hline Gene & Primer & Primer sequence & $\begin{array}{c}\text { Size of PCR } \\
\text { product }\end{array}$ \\
\hline REV & F & TTGTTGAAGGCAAGCATCAG & $105 \mathrm{bp}$ \\
& R & GAGGATAGCATCTGCCCTTT & \\
ALV-J & F & TGCGTGCGTGGTTATATTTC & $144 \mathrm{bp}$ \\
& R & AATGGTGAGGTCGCTGACTGT & \\
GADPH & F & GAACATCATCCCAGCGTCCA & $132 \mathrm{bp}$ \\
& R & CGGCAGGTCAGGTCAACAAC & \\
\hline
\end{tabular}

\section{Target prediction}

miRanda (http://www.microrna.org/microrna/) was used to predict targets of the differentially expressed miRNA. Gene enrichment and functional annotation analyses were conducted using Gene Ontology (GO; www.geneo ntology.org), Kyoto Encyclopedia of Genes and Genomes (KEGG, http://www.genome.jp/kegg), PATHWAY Database and LIGAND Database.

\section{Statistical analysis}

Results are presented as the mean \pm standard deviation(s). The $\mathrm{T}$ test and one-way ANOVA test was performed using SPSS 13.0 statistical software. A P value less than 0.05 was considered statistically significant.

\section{Additional files}

Additional file 1. The potential miRNA targets of the differentially expressed miRNA between ExoRJ and ExoJ. Numerous target genes, 19,450 for 54 miRNAs, were predicted as potential miRNA targets.

Additional file 2. The potential miRNA targets of the differentially expressed miRNA between ExoRJ and ExoR. Numerous target genes, 6058 for 16 miRNAs, were predicted as potential miRNA targets.

Additional file 3. The $\mathrm{GO}$ annotation of the predicted target genes of the differentially expressed miRNA between ExoRJ and ExoJ. A GO annotation of the predicted target genes revealed that 100 target genes were annotated significantly for the 54 differentially expressed miRNAs between ExoRJ and ExoJ.

Additional file 4. The $\mathrm{GO}$ annotation of the predicted target genes of the differentially expressed miRNA between ExoRJ and ExoR. A GO annotation of the predicted target genes revealed that 35 target genes were annotated significantly for the 16 miRNAs between ExoRJ and ExoR.

Additional file 5. The KEGG analysis of the predicted target genes of the differentially expressed miRNA between ExoRJ and ExoJ. The KEGG analysis of the predicted target genes revealed that 7 regulatory networks were annotated significantly for the 54 differentially expressed miRNAs between ExoRJ and ExoJ.

Additional file 6. The KEGG analysis of the predicted target genes of the differentially expressed miRNA between ExoRJ and ExoR. The KEGG analysis of the predicted target genes revealed that 3 regulatory networks were annotated significantly for the 16 differentially expressed miRNAs between ExoRJ and ExoR.

\section{Authors' contributions}

ZC conceived and designed the research. DZ wrote the manuscript. DZ and JW performed the experiments. SH, XD and JZ contributed reagents and materials. $C L, L H, V N$, and $Y Y$ commented on the manuscript. All authors read and approved the final manuscript.

\section{Author details \\ ${ }^{1}$ College of Veterinary Medicine, Shandong Agricultural University, Tai'an 271018, China. ${ }^{2}$ The Pirbright Institute \& UK-China Centre of Excellence on Avian Disease Research, Pirbright, Ash Road, Guildford, Surrey GU24 ONF, UK. \\ Acknowledgements \\ We are grateful to Ms. Pingping Zhuang, Dr. Guihua Wang, Ms. Li Zhang and the Annoroad Gene Technology Company for their technical assistance. We thank Dr. Gen Li and Dr. Mingjun Zhu for their helpful discussion and manu- script revision.}




\section{Competing interests}

The authors declare no competing financial interests.

\section{Availability of data and materials}

The datasets used and/or analysed during the current study are available from the corresponding author on reasonable request.

\section{Consent for publication}

All authors consented to the publication of this manuscript. The funding agencies had no role in the decision to publish this manuscript.

\section{Ethics approval and consent to participate}

Approval for this study was obtained through the College of Veterinary Medicine of Shandong Agricultural University.

\section{Funding}

The study was supported by grants from the China-UK Partnership on Global Food Security: Combating tumour diseases for Sustainable Poultry Production (31761133002, BB/R012865/1), the Natural Science Foundation of China (31672521), the Shandong Modern Agricultural Technology \& Industry System (SDAIT-11-04) and the Fund of Shandong "Double Tops" Program (2017).

\section{Publisher's Note}

Springer Nature remains neutral with regard to jurisdictional claims in published maps and institutional affiliations.

Received: 14 March 2018 Accepted: 21 June 2018

Published online: 03 July 2018

\section{References}

1. Payne LN, Brown SR, Bumstead N, Howes K, Frazier JA, Thouless ME. A novel subgroup of exogenous avian leukosis virus in chickens. J Gen Virol. 1991;72(Pt 4):801-7.

2. Bai J, Payne LN, Skinner MA. HPRS-103 (exogenous avian leukosis virus, subgroup J) has an env gene related to those of endogenous elements EAV-0 and E51 and an E element found previously only in sarcoma viruses. J Virol. 1995;69:779-84

3. Dong X, Ju S, Zhao P, Li Y, Meng F, Sun P, Cui Z. Synergetic effects of subgroup J avian leukosis virus and reticuloendotheliosis virus co-infection on growth retardation and immunosuppression in SPF chickens. Vet Microbiol. 2014;172:425-31.

4. Guo H, Li H, Cheng Z, Liu J, Cui Z. Co-Infection on immunologic function of T lymphocytes and histopathology in broiler chickens. J Integr Agric. 2010;09:1667-76.

5. Cui Z, Sun S, Zhang Z, Meng S. Simultaneous endemic infections with subgroup J avian leukosis virus and reticuloendotheliosis virus in commercial and local breeds of chickens. Avian Pathol. 2009;38:443-8.

6. Davidson I, Borenstein R. Multiple infection of chickens and turkeys with avian oncogenic viruses: prevalence and molecular analysis. Acta Virol. 1999;43:136-42

7. Cheng Z, Zhang H, Wang G, Liu Q, Liu J, Guo H, Zhou E. Investigations of avian leukosis virus subgroup $J$ and reticuloendotheliosis virus infections in broiler breeders in China. Isr J Vet Med. 2011:66:34-8.

8. Harding C, Heuser J, Stahl P. Receptor-mediated endocytosis of transferrin and recycling of the transferrin receptor in rat reticulocytes. J Cell Biol. 1983;97:329-39.

9. Admyre C, Johansson SM, Qazi KR, Filen JJ, Lahesmaa R, Norman M, Neve EP, Scheynius A, Gabrielsson S. Exosomes with immune modulatory features are present in human breast milk. J Immunol. 2007;179:1969-78.

10. Masyuk Al, MasyukTV, Larusso NF. Exosomes in the pathogenesis, diagnostics and therapeutics of liver diseases. J Hepatol. 2013;59:621-5.

11. Vella $\cup$, Sharples RA, Nisbet RM, Cappai R, Hill AF. The role of exosomes in the processing of proteins associated with neurodegenerative diseases. Eur Biophys J. 2008:37:323-32

12. Bard MP, Hegmans JP, Hemmes A, Luider TM, Willemsen R, Severijnen LA, van Meerbeeck JP, Burgers SA, Hoogsteden HC, Lambrecht BN. Proteomic analysis of exosomes isolated from human malignant pleural effusions. Am J Respir Cell Mol Biol. 2004;31:114-21.

13. Schorey JS, Bhatnagar S. Exosome function: from tumor immunology to pathogen biology. Traffic. 2008;9:871-81.

14. Beach A, Zhang HG, Ratajczak MZ, Kakar SS. Exosomes: an overview of biogenesis, composition and role in ovarian cancer. J Ovarian Res. 2014;7:14

15. El-Saghir J, Nassar F, Tawil N, El-Sabban M. ATL-derived exosomes modulate mesenchymal stem cells: potential role in leukemia progression. Retrovirology. 2016;13:73.

16. Milane L, Singh A, Mattheolabakis G, Suresh M, Amiji MM. Exosome mediated communication within the tumor microenvironment. J Control Release. 2015:219:278-94

17. Greening DW, Gopal SK, Xu R, Simpson RJ, Chen W. Exosomes and their roles in immune regulation and cancer. Semin Cell Dev Biol. 2015;40:72-81.

18. Narayanan A, Jaworski E, Duyne RV, lordanskiy S, Guendel I, Das R, Currer R, Sampey G, Chung M, Kehn-Hall K. Exosomes derived from HTLV-1 infected cells contain the viral protein Tax. Retrovirology. 2014;11:046.

19. Selbach M, Schwanhausser B, Thierfelder N, Fang Z, Khanin R, Rajewsky N. Widespread changes in protein synthesis induced by microRNAs. Nature. 2008:455:58-63.

20. Baek D, Villen J, Shin C, Camargo FD, Gygi SP, Bartel DP. The impact of microRNAs on protein output. Nature. 2008:455:64-71.

21. Shin WY, Chu KM. MiRNA as potential biomarkers and therapeutic targets for gastric cancer. World J Gastroenterol. 2014;20:10432-9.

22. Lytle JR, Yario TA, Steitz JA. Target mRNAs are repressed as efficiently by microRNA-binding sites in the 5'UTR as in the 3'UTR. Proc Natl Acad Sci USA. 2007:104:9667-72.

23. Towler BP, Jones Cl, Newbury SF. Mechanisms of regulation of mature miRNAs. Biochem Soc Trans. 2015:43:1208-14.

24. Ambros V. MicroRNAs and developmental timing. Curr Opin Genet Dev. 2011;21:511-7.

25. Blahna MT, Hata A. Regulation of miRNA biogenesis as an integrated component of growth factor signaling. Curr Opin Cell Biol. 2013;25:233-40.

26. Subramanian S, Steer CJ. MicroRNAs as gatekeepers of apoptosis. J Cell Physiol. 2010;223:289-98.

27. Zhou R, Rana TM. RNA-based mechanisms regulating host-virus interactions. Immunol Rev. 2013;253:97-111.

28. Bhaskaran M, Mohan M. MicroRNAs: history, biogenesis, and their evolving role in animal development and disease. Vet Pathol. 2014;51:759-74.

29. Hartig SM, Hamilton MP, Bader DA, McGuire SE. The miRNA interactome in metabolic homeostasis. Trends Endocrinol Metab. 2015;26:733-45.

30. Rosado J, Alvarez C, Clark D, Gotuzzo E, Talledo M. Differential miRNA expression profiles in Peruvian HTLV-1 carriers. Retrovirology. 2014;11:1.

31. Gupta P, Liu B, Wu JQ, Soriano V, Vispo E, Carroll AP, Goldie BJ, Cairns MJ, Saksena NK. Genome-wide mRNA and miRNA analysis of peripheral blood mononuclear cells (PBMC) reveals different miRNAs regulating HIV/HCV co-infection. Virology. 2014:450-451:336-49.

32. Janssen HL, Reesink HW, Lawitz EJ, Zeuzem S, Rodriguez-Torres M, Patel K, van der Meer AJ, Patick AK, Chen A, Zhou Y, et al. Treatment of HCV infection by targeting microRNA. N Engl J Med. 2013;368:1685-94

33. Pilotti E Casoli C, Bianchi MV Bignami F Prati F. MiRNA profile in CD4 positive T cells from HTLV-2 and HIV-1 mono- and co-infected subjects. Retrovirology. 2012;9:P2.

34. Quinlan AR, Hall IM. BEDTools: a flexible suite of utilities for comparing genomic features. Bioinformatics. 2010;26:841-2.

35. Cho JA, Yeo DJ, Son HY, Kim HW, Jung DS, Ko JK, Koh JS, Kim YN, Kim CW. Exosomes: a new delivery system for tumor antigens in cancer immunotherapy. Int J Cancer. 2005;114:613-22.

36. Hegmans JP, Bard MP, Hemmes A, Luider TM, Kleijmeer MJ, Prins JB, Zitvogel L, Burgers SA, Hoogsteden HC, Lambrecht BN. Proteomic analysis of exosomes secreted by human mesothelioma cells. Am J Pathol. 1807;2004:164.

37. Lamparski HG, Metha-Damani A, Yao JY, Patel S, Hsu DH, Ruegg C, Le PJ. Production and characterization of clinical grade exosomes derived from dendritic cells. J Immunol Methods. 2002;270:211-26.

38. Schorey JS, Cheng Y, Singh PP, Smith VL. Exosomes and other extracellular vesicles in host-pathogen interactions. EMBO Rep. 2015;16:24-43.

39. Fleming A, Sampey G, Chung MC, Bailey C, van Hoek ML, Kashanchi F, Hakami RM. The carrying pigeons of the cell: exosomes and their role in infectious diseases caused by human pathogens. Pathog Dis. 2014:71:109-20 
40. Taylor DD, Zacharias W, Gerceltaylor C. Exosome isolation for proteomic analyses and RNA profiling. Methods Mol Biol. 2011;728:235.

41. Yang G, Zhang W, Yu C, Ren J, An Z. MicroRNA let-7: regulation, single nucleotide polymorphism, and therapy in lung cancer. J Cancer Res Ther. 2015;11(Suppl 1):C1-6.

42. Minemura H, Takagi K, Miki Y, Shibahara Y, Nakagawa S, Ebata A, Watanabe M, Ishida T, Sasano H, Suzuki T. Abnormal expression of miR-1 in breast carcinoma as a potent prognostic factor. Cancer Sci. 2015;106:1642-50.

43. Porrello ER, Johnson BA, Aurora AB, Simpson E, Nam YJ, Matkovich SJ, Dorn GW 2nd, van Rooij E, Olson EN. MiR-15 family regulates postnatal mitotic arrest of cardiomyocytes. Circ Res. 2011;109:670-9.
44. Pekarsky Y, Croce CM. Role of miR-15/16 in CLL. Cell Death Differ. 2015;22:6-11.

45. Kramer LD. Complexity of virus-vector interactions. Curr Opin Virol. 2016;21:81-6.

46. Hirschey MD, DeBerardinis RJ, Diehl AME, Drew JE, Frezza C, Green MF, Jones LW, Ko YH, Le A, Lea MA, et al. Dysregulated metabolism contributes to oncogenesis. Semin Cancer Biol. 2015;35(Suppl):S129-50.

47. Martinez-Outschoorn UE, Lisanti MP, Sotgia F. Catabolic cancer-associated fibroblasts transfer energy and biomass to anabolic cancer cells, fueling tumor growth. Semin Cancer Biol. 2014;25:47-60.

48. Wang Y, Wang G, Wang Z, Zhang H, Zhang L, Cheng Z. Chicken biliary exosomes enhance CD4(+)T proliferation and inhibit ALV-J replication in liver. Biochem Cell Biol. 2014;92:145-51.
Ready to submit your research? Choose BMC and benefit from:

- fast, convenient online submission

- thorough peer review by experienced researchers in your field

- rapid publication on acceptance

- support for research data, including large and complex data types

- gold Open Access which fosters wider collaboration and increased citations

- maximum visibility for your research: over $100 \mathrm{M}$ website views per year

At BMC, research is always in progress.

Learn more biomedcentral.com/submissions 